REF - ISSN 1808-0804 Vol. VI (1), 33 - 43, 2009

\title{
ESTUDO DOS MEDICAMENTOS UTILIZADOS PELOS PACIENTES ATENDIDOS EM LABORATÓRIO DE ANÁLISES CLÍNICAS E SUAS INTERFERÊNCIAS EM TESTES LABORATORIAIS: UMA REVISÃO DA LITERATURA
}

\author{
Profile of medicines used for outpatients assisted at clinical analysis laboratory and the \\ interference in laboratory tests:a literature review
}

\author{
Bárbara C. Ferreira ${ }^{1,2^{*}}$; Karina L. dos Santos ${ }^{1,3}$; Steph C. Rudolph ${ }^{1,3}$; \\ Joana D. X. Alcanfor ${ }^{1,4}$; Luiz C. da Cunha ${ }^{1,5}$ \\ ${ }^{1}$ Faculdade de Farmácia, Universidade Federal de Goiás. Praça Universitária s/n. 74605-220, Goiânia-GO, Brasil. \\ ${ }^{2}$ Graduanda do curso de Farmácia FF/UFG e graduanda do curso de Química Industrial CEFET/GO. \\ ${ }^{3}$ Graduando (a) do curso de Farmácia FF/UFG. \\ ${ }^{4}$ Professora de Bioquímica Clínica e Coordenadora do Laboratório Rômulo Rocha, FF-UFG; Co-Orientadora. \\ ${ }^{5}$ Prof. de Toxicologia e Farmacocinética e Coordenador do Núcleo de Estudos e Pesquisas Tóxico-Farmacológicas \\ da UFG (NEPET-UFG); Orientador.
}

* Autor para correspondência e-mail: babibs12@hotmail.com

Recebido em 01/07/2008 - Aceito em 05/09/2008

RESUMO: A interferência de medicamentos em análises clínicas assume importante papel na rotina laboratorial pela probabilidade de interferir nos ensaios e modificar o diagnóstico clínico-laboratorial. Muitos fármacos exercem efeitos in vivo, in vitro ou ambos simultaneamente sobre os testes laboratoriais. O objetivo do presente trabalho foi identificar os medicamentos mais usados pelos pacientes que foram atendidos pelo Centro de Análises Clínicas Rômulo Rocha em Goiânia (GO) no período de junho a dezembro de 2007 e analisar possíveis interações entre estes medicamentos e exames laboratoriais. Para isso foi traçado um estudo descritivo e retrospectivo, através da análise de 600 fichas dos medicamentos mais utilizados pelos pacientes e usuários dos serviços prestados pelo laboratório. Dentre os medicamentos pesquisados foram selecionados os cinco mais utilizados para que fosse feita uma discussão a respeito de suas interações em testes laboratoriais. Os cinco medicamentos mais utilizados foram: captopril, enalapril, hidroclorotiazida, levotiroxina sódica e propranolol. Dentre os cinco medicamentos verificou-se maior interferência in vivo do que in vitro. Como exemplo dessas interferências podemos citar: o captopril que pode proporcionar resultados falso-positivos em cetonas na urina; o enalapril que pode interferir no fator antinúcleo positivamente; a hidroclorotiazida que aumenta a concentração da uréia in vivo; a levotiroxina sódica que pode provocar redução por efeito fisiológico no exame de apolipoproteína B e o propranolol que pode provocar níveis elevados de uréia sanguínea. Os resultados obtidos podem, também, ser utilizados para promoção e planejamento de ações de Atenção Farmacêutica.

PALAVRAS-CHAVE: testes laboratoriais, interações de medicamentos, farmacoepidemiologia.

ABSTRACT: The drugs interference in clinical analysis takes an important role in routine laboratory for interfering in trials and modifies the clinical and laboratory diagnosis. Many drugs exert effects in vivo, in vitro or both simultaneously on the laboratory tests. The objective of this study was to identify the most used drugs for patients who were assisted at the Centro de Análises Clínicas Rômulo Rocha in Goiânia (GO) in the period from June to December 2007 and examining possible interactions between the most used drugs for patients and laboratory tests. For this route was a descriptive and retrospective study, through the analysis of 600 sheets, most of the drugs used for patients and service users provided by the laboratory. Among the drugs surveyed, were selected 
the five most used to be made a discussion about your interactions in laboratory tests. The five drugs most used were: captopril, enalapril, hydrochlorothiazide, levotiroxine sodium and propranolol. Among the five medicines there was more interference in vivo than in vitro. As an example of such interference can cite: the captopril, which can provide false-positive results in ketones in the urine; enalapril that may interfere with antinuclear antibody, the hydrochlorothiazide which increases the concentration of urea in vivo; tyroxine the sodium that can cause a reduction physiological effect in the examination of apolipoprotein $B$ and propranolol that can cause high levels of blood urea. The results can also be used for promotion and planning of actions of Pharmaceutical Care.

KEYWORDS: laboratory test, drug interactions, pharmacoepidemiology.

\section{INTRODUÇÃO}

A farmacovigilância foi definida pelo Conselho Federal de Farmácia (CFF), na resolução $n^{\circ}$. 357 de 2001, como a identificação e avaliação dos efeitos, agudos ou crônicos, do risco do uso dos tratamentos farmacológicos no conjunto das populações ou em grupos de pacientes expostos a tratamentos específicos.

O presente trabalho tem como base os temas farmacovigilância e farmacoepidemiologia visando demonstrar a importância desses assuntos quando relacionados ao uso de medicamentos e realização de exames laboratoriais. A população escolhida para o levantamento dos fármacos mais utilizados foram os usuários do Centro de Análises Clínicas Rômulo Rocha.

O Centro de Análises Clínicas Rômulo Rocha foi fundado em maio de 1971 em Goiânia - Goiás com o objetivo de desenvolvimento de estágio supervisionado do curso de Farmácia da Universidade Federal de Goiás. Realiza exames complementares nas áreas de microbiologia, micologia, citopatologia, hematologia, parasitologia, bioquímica, uranálise, sorologia, hormônios, marcadores tumorais e toxicologia (FACULDADE DE FARMÁCIA UFG, 2008).

O levantamento epidemiológico de fármacos, utilizados por uma população específica, pode ser importante para auxiliar na prevenção do surgimento de problemas relacionados aos medicamentos, PRM (PEREIRA et al., 2004), incluindo as interferências entre os fármacos e os resultados de análises laboratoriais.

Recentes publicações revelam que aproximadamente 68 a 93\% dos erros laboratoriais encontrados são conseqüência da falta de padronização na fase pré-analítica. Portanto, é de extrema importância implementar metodologias mais rigorosas para detecção, classificação e redução destes erros. A fase pré-analítica consiste na preparação do paciente, coleta, manipulação e armazenamento da amostra antes da determinação analítica, ou seja, compreende todas as etapas que precedem ao ensaio laboratorial, dentro ou fora do laboratório de análises clínicas e toxicológicas (FERREIRA et al., 2007).

A influência das variáveis pré-analíticas pode tornar-se desprezível, desde que se estabeleça uma boa orientação aos pacientes em relação ao jejum adequado, à não realização de exercícios físicos extenuantes no período que antecede a coleta do material biológico, a informações relativas ao hábito de fumar e relativas ao período do ciclo menstrual em que a cliente se encontra. Também é muito importante obter informações sobre a utilização de fármacos e/ou drogas terapêuticas que eventualmente estejam em uso (FERREIRA et al., 2007).

A interferência de medicamentos em análises clínicas assume importante papel na rotina laboratorial por interferir nos ensaios e modificar o diagnóstico clínico-laboratorial (MARTINELLO e SILVA, 2003). Muitos fármacos exercem efeitos in vivo, in vitro ou ambos simultaneamente sobre os testes laboratoriais. Quando um medicamento induz mudança de um parâmetro biológico através de um mecanismo fisiológico ou farmacológico, tem-se a interferência in vivo ou reação adversa do organismo ao medicamento. Por outro lado, por interferência puramente analítica do fármaco ou de seu catabólito, pode, em alguma etapa analítica, interagir com as substâncias constituintes dos reagentes químicos utilizados, causando um falso resultado da análise. Essa reação indesejada é conhecida como interferência in vitro ou analítica (MOTTA, 2003).

Muitas são as drogas interferentes em exames laboratoriais, tanto in vitro quanto in vivo, sendo as últimas também denominadas reações adversas a medicamentos (GIACOMELLI e PEDRAZZI, 2001). Um exemplo de interferência analítica é o falso aumento dos valores de frutosamina no soro por pacientes utilizando o captopril. Como exemplo de interferência por efeito fisiológico pode-se citar o enalapril e a hidroclorotiazida que causam alterações nas dosagens de ácido úrico no soro. Outra interferência por efeito fisiológico é observada na utilização do propranolol e/ou levotiroxina na realização do exame de tiroxina (T4) livre no soro (YOUNG, 1995).

As doenças cardiovasculares são as principais causas de morte da população adulta dos países desenvolvidos. O Brasil está sofrendo uma transição epidemiológica que significa a mudança na incidência das causas de mortalidade, passando de causas infecto-contagiosas e maternas, as mais incidentes, para doenças crônico degenerativas, como a hipertensão arterial sistêmica (TRINDADE et al., 1998). Portanto, a hipertensão consiste em um grande problema de saúde pública e neste estudo dentre os fármacos mais utilizados, quatro deles (captopril, enalapril, hidroclorotiazida e propranolol) são utilizados para o tratamento da hipertensão arterial.

O hipotireoidismo é a alteração da função tireoidiana mais freqüente e geralmente de diagnóstico e tratamento simples. Tem como causa mais comum a tireoidite auto-imune em todas as suas formas de apresentação. O tratamento do hipotireoidismo consiste na reposição de hormônio tireoidiano, em geral com 
levotiroxina sódica (SILVA e SOUZA, 2005) que foi também um dos fármacos mais utilizados dentre os pesquisados no trabalho.

O objetivo do presente trabalho é identificar os medicamentos mais usados pelos pacientes que foram atendidos pelo Centro de Análises Clínicas Rômulo Rocha no período de junho a dezembro de 2007 e analisar possíveis interações entre os medicamentos mais utilizados pelos pacientes e os exames laboratoriais. Tem-se também uma preocupação em abordar os temas relacionados à farmacoepidemiologia e à farmacovigilância.

\section{MATERIAL E MÉTODOS}

Foi traçado um estudo descritivo e retrospectivo dos fármacos mais utilizados pelos pacientes e usuários dos serviços prestados pelo Centro de Análises Clínicas Rômulo Rocha em Goiânia (GO). Os dados foram coletados através de análise de cadastros e fichas com informações pessoais dos pacientes. Estas informações já haviam sido coletadas e registradas durante o cadastro do paciente no laboratório, não sendo necessário a realização de entrevistas.

A população estudada abrange pessoas de ambos sexos, independente de faixa etária, cor, classe ou grupo social. Foram analisadas 600 fichas de pacientes atendidos no período de junho a dezembro de 2007. Inicialmente o período escolhido para análise de dados era de janeiro a dezembro de 2007, entretanto o laboratório só começou a indagar e arquivar os medicamentos utilizados pelos pacientes a partir do mês de junho de 2007. O levantamento dos dados iniciou-se em março de 2008 selecionando-se cerca de 85 fichas que continham relatos do uso de fármacos, em cada mês pesquisado, de forma que a amostra representasse todo o período.

As fichas e cadastros, juntamente com os resultados dos exames laboratoriais realizados pelos pacientes, ficam armazenados no próprio laboratório.

Coletaram-se os seguintes dados: número do protocolo de registro do paciente no laboratório, iniciais dos nomes dos pacientes, idade, sexo, fármacos utilizados e exames laboratoriais realizados. A identidade do paciente permaneceu em sigilo, visto que não se utilizou o nome completo, adotando-se como sigla apenas as suas iniciais, sendo identificado também através do número de protocolo. A pesquisa foi previamente aprovada pelo comitê de ética da Universidade Federal de Goiás - UFG.

Após a coleta dos dados, os fármacos foram contados, relacionados e agrupados em classes terapêuticas. Escolheram-se os cinco fármacos mais utilizados pelos pacientes para a pesquisa da interferência dos mesmos em exames laboratoriais. Os medicamentos foram relacionados de acordo com seus princípios ativos pesquisados na literatura.

\section{RESULTADOS E DISCUSSÃO}

Dentre as 600 fichas pesquisadas, 421 delas eram referentes ao sexo feminino e 179 ao sexo masculino conforme tabela 1. Além das diferenças hormonais específicas e características de cada sexo, outros parâmetros sangüíneos e urinários se apresentam em concentrações distintas entre homens e mulheres em decorrência das diferenças metabólicas. Em geral, os intervalos de referência para estes parâmetros são específicos para cada gênero (SUMITA et al., 2005).

Tabela 1. Relação da porcentagem de pacientes em relação ao gênero

\begin{tabular}{c|c|c}
\hline Gênero & Quantidade & Porcentagem (\%) \\
\hline Masculino & 179 & 29,83 \\
\hline Feminino & 421 & 70,17 \\
\hline Total & 600 & 100 \\
\hline
\end{tabular}

Alguns parâmetros bioquímicos possuem concentração sérica dependente da idade do indivíduo. Esta dependência é resultante de diversos fatores, como maturidade funcional dos órgãos e sistemas, conteúdo hídrico e massa corporal. Em situações específicas, até os intervalos de referência devem considerar essas diferenças. É importante lembrar que as mesmas causas de variações pré-analíticas, que afetam os resultados laboratoriais em indivíduos jovens, interferem nos resultados dos exames realizados em indivíduos idosos, mas a intensidade da variação tende a ser maior neste grupo etário. Doenças subclínicas também são mais comuns nos idosos e precisam ser consideradas na avaliação da variabilidade dos resultados, ainda que as próprias variações biológicas e ambientais não devam ser subestimadas (SUMITA et al., 2005). Na tabela 2 estão relacionadas as idades, por faixa etária, dos pacientes e as porcentagens referentes as mesmas. 
Tabela 2. Relação das idades por faixa etária dos pacientes com suas respectivas porcentagens

\begin{tabular}{c|c|c|c}
\hline Faixa etária & Quantidade de pacientes & Quantidade (\%) & Quantidade de fármacos \\
\hline 0 a 10 & 23 & 3,8 & 30 \\
\hline 11 a 20 & 39 & 6,5 & 82 \\
\hline 21 a 30 & 51 & 8,5 & 94 \\
\hline 31 a 40 & 75 & 12,5 & 241 \\
\hline 41 a 50 & 114 & 19 & 315 \\
\hline 51 a 60 & 143 & 23,8 & 228 \\
\hline 61 a 70 & 85 & 14,2 & 146 \\
\hline 71 a 80 & 56 & 9,3 & 48 \\
\hline 81 a 91 & 14 & 2,4 & 1344 \\
\hline Total & 600 & 100 &
\end{tabular}

Durante o período estudado, foram relatados 197 princípios ativos diferentes, sendo que alguns fármacos foram relatados por mais de um paciente e alguns pacientes relataram uso de mais de um fármaco. Também foram encontrados 172 medicamentos grafados de forma incorreta e/ou não foram encontrados na literatura (exemplos: Redevite, Anatilix, Glicorazida, Desconjequison, etc.), totalizando 1344. Para melhor visualização, os princípios ativos foram agrupados de acordo com sua classe terapêutica conforme Tabela 3. Os fármacos foram subdivididos conforme as classes terapêuticas encontradas no Dicionário de Especialidades Farmacêuticas (2005) e no Bulário Eletrônico da ANVISA (2008). A representação da porcentagem de fármacos relatados erroneamente/inexistentes está representada na Figura 1.

Tabela 3. Classes terapêuticas dos fármacos relatados pelos pacientes nas fichas analisadas no período de junho a dezembro de 2007.

\begin{tabular}{c|c|c|c}
\hline $\begin{array}{c}\text { Classes } \\
\text { terapêuticas }\end{array}$ & $\begin{array}{c}\text { Sub-classes } \\
\text { terapêuticas }\end{array}$ & $\begin{array}{c}\text { Quantidade de } \\
\text { fármacos }\end{array}$ & Fármacos citados \\
\hline $\begin{array}{c}\text { Analgésicos, } \\
\begin{array}{c}\text { antiinflamatórios e } \\
\text { antipiréticos }\end{array}\end{array}$ & $\begin{array}{c}\text { Analgésicos e } \\
\text { antiinflamatórios } \\
\text { não-esteroidais (AINES) } \\
\text { e antipiréticos }\end{array}$ & 100 & $\begin{array}{c}\text { acetaminofeno (paracetamol), ácido } \\
\text { acetilsalicílico, ácido mefenâmico, carisoprodol + } \\
\text { paracetamol + fenilbutazona, diclofenaco de sódio } \\
\text { + codeína, dipirona sódica, morfina (sulfato de), } \\
\text { paracetamol, paracetamol + carisoprodol + } \\
\text { cafeína, paracetamol + codeína, celocoxib, } \\
\text { cetoprofeno, diclofenaco de sódio, etodolaco, } \\
\text { ibuprofeno, meloxicam, naproxeno sódico, } \\
\text { nimesulida, piroxicam, tenoxicam }\end{array}$ \\
\cline { 2 - 4 } & Corticosteróides & 36 & $\begin{array}{c}\text { budesonida, dexametasona, formoterol (fumarato } \\
\text { de) + budesonida, hidrocortisona, prednisolona, } \\
\text { prednisona }\end{array}$ \\
\hline
\end{tabular}

\begin{tabular}{|c|c|c|c|}
\hline \multirow[t]{5}{*}{$\begin{array}{l}\text { Fármacos que } \\
\text { atuam no SNC }\end{array}$} & Ansiolíticos & 21 & cloxazolam, diazepam, lorazepam, nitrazepam \\
\hline & Anticonvulsivantes & 47 & $\begin{array}{l}\text { carbamazepina, clonazepam, divalproato de } \\
\text { sódio, fenitoína, fenobarbital, gabapentina, } \\
\text { oxcarbamazepina }\end{array}$ \\
\hline & Antidepressivos & 31 & $\begin{array}{c}\text { amitriptilina (cloridrato de), citalopam, fluoxetina, } \\
\text { imipramina, nortriptilina, sertralina (cloridrato de), } \\
\text { trazodona }\end{array}$ \\
\hline & Antipsicóticos & 11 & $\begin{array}{l}\text { lítio (carbonato de), quetiapina (fumarato de), } \\
\text { tioridazina (cloridrato de) }\end{array}$ \\
\hline & Antieméticos & 14 & $\begin{array}{l}\text { dimenidrato, domperidona, meclizina (cloridrato } \\
\text { de), metoclopramida (cloridrato de), talidomida }\end{array}$ \\
\hline \multirow[t]{2}{*}{$\begin{array}{c}\text { Fármacos } \\
\text { cardiovasculares }\end{array}$} & Antiarrítmicos & 16 & $\begin{array}{c}\text { amiodarona (cloridrato de), metoprolol (succinato } \\
\text { de), verapamil (cloridrato de) }\end{array}$ \\
\hline & Anti-hipertensivos & 302 & $\begin{array}{c}\text { anlodipina (besilato), atenolol, candesartana } \\
\text { cilexetila, captopril, carvedilol, clonidina (cloridrato } \\
\text { de), diltiazem (cloridrato de), doxazosina (mesilato } \\
\text { de), enalapril, hidralazina (cloridrato de), } \\
\text { indapamina, lisinopril, losartana potássica, } \\
\text { losartana potássica + hidroclotiazida, metildopa, }\end{array}$ \\
\hline
\end{tabular}




\begin{tabular}{|c|c|c|c|}
\hline & & & $\begin{array}{c}\text { propranolol (cloridrato de), propranolol + } \\
\text { hidroclorotiazida }\end{array}$ \\
\hline & Antilipêmicos & 23 & $\begin{array}{l}\text { ciprofibrato, genfibrozila, rosuvastatina cálcica, } \\
\text { sinvastatina }\end{array}$ \\
\hline & Cardiotônicos & 10 & digoxina, betametildigoxina \\
\hline & Vasodilatadores & 34 & $\begin{array}{c}\text { cilostazol, ginkgo biloba, monocordil, nifedipina, } \\
\text { propatilnitrato }\end{array}$ \\
\hline & Diuréticos & 110 & $\begin{array}{l}\text { bumetanida, espironolactona, furosemida, } \\
\text { hidroclorotiazida, hidroclorotiazida + amilorida } \\
\text { (cloridrato de) }\end{array}$ \\
\hline \multirow{2}{*}{$\begin{array}{l}\text { Fármacos que } \\
\text { agem no TGl }\end{array}$} & Antidiarréicos & 24 & Saccharomyces cerevisiae \\
\hline & Antiulcerosos & 28 & $\begin{array}{c}\text { famotidina, lansoprazol, omeprazol, ranitidina } \\
\text { (cloridrato) }\end{array}$ \\
\hline Anti-histamínicos & Anti-histamínicos & 11 & $\begin{array}{l}\text { ciproeptadina (cloridrato de), dexclorfeniramina, } \\
\text { hidroxizina, loratadina, prometazina (cloridrato de) }\end{array}$ \\
\hline Antidiabéticos & $\begin{array}{l}\text { Hipoglicemiantes orais, } \\
\text { Insulina }\end{array}$ & 59 & $\begin{array}{l}\text { glibenclamida, glicazida, glimepirida, metformina } \\
\text { (cloridrato de), insulina }\end{array}$ \\
\hline Antimicrobianos & $\begin{array}{c}\text { Antibióticos, } \\
\text { antifúngicos, } \\
\text { antisséptico urinário, } \\
\text { anti-retrovirais, } \\
\text { quimioterápicos }\end{array}$ & 55 & $\begin{array}{c}\text { amoxicilina, azitromicina, } \\
\text { benzilpenicilina/benzatina, ciprofloxacino, } \\
\text { eritromicina, lamivudina + zidovudina, lansoprazol } \\
\text { + claritromicina + amoxicilina, levofloxacino, } \\
\text { limeciclina, metenamina + metiltionínio, } \\
\text { nitrofurantoína, norfloxacino, polimixina B + } \\
\text { neomicina + hidrocortisona, ribavirina, } \\
\text { sulfametoxazol + trimetoprima + } \\
\text { fenilazodiaminopiridina (cloridrato de), } \\
\text { sulfametoxazol + trimetoprima, tirotricina, } \\
\text { zidovudina }\end{array}$ \\
\hline
\end{tabular}

\begin{tabular}{|c|c|c|c|}
\hline \multirow[t]{2}{*}{ Hormônios } & $\begin{array}{l}\text { Hormônios } \\
\text { Femininos } \\
\text { Conjugados }\end{array}$ & 12 & $\begin{array}{c}\text { ciproterona (acetato de) + etinilestradiol, } \\
\text { clordiazepóxido (cloridrato de) + estrógenos, } \\
\text { estradiol (valerato de) + ciproterona (acetato de), } \\
\text { estrogênio, estrogênio conjugado + } \\
\text { medroxiprogesterona (acetato de), gestodeno + } \\
\text { etinilestradiol, noretisterona (acetato de) + } \\
\text { estradiol }\end{array}$ \\
\hline & Hormônios tireoidianos & 89 & levotiroxina sódica \\
\hline \multirow{2}{*}{$\begin{array}{l}\text { Fármacos que } \\
\text { agem no sangue }\end{array}$} & Anticoagulantes & 12 & femprocumona, heparina sódica, warfarin sódica \\
\hline & Antianêmicos & 33 & $\begin{array}{l}\text { ácido fólico, alfaepoetina, eritropoetina, hidreia, } \\
\text { hidróxido de ferro polimaltosado, sulfato ferroso }\end{array}$ \\
\hline Recalcificantes & Recalcificantes & 13 & $\begin{array}{l}\text { carbonato de cálcio, raloxifeno, alendronato de } \\
\text { sódio }\end{array}$ \\
\hline \multirow[t]{2}{*}{ Vitaminas } & Vitaminas & 21 & $\begin{array}{c}\text { ácido ascórbico, calciferol (vitamina D), calcitriol, } \\
\text { cobalamina (vitamina B 12), complexo vitamínico } \\
\text { B, retinol (vitamina A) }\end{array}$ \\
\hline & & 60 & $\begin{array}{l}\text { ácido hidroxicítrico + crômio, ácido valpróico, } \\
\text { alfapeginterferona, aminofilina, azatioprina, } \\
\text { baclofeno, betametasona, biperideno (cloridrato } \\
\text { de), bromazepan, bromocriptina, bromoprida, } \\
\text { bronfeniramina (maleato de) + fenilefrina } \\
\text { (cloridrato de), bronfeniramina (maleato de) + } \\
\text { fenilefrina (cloridrato de) + fenilpropanolamina', } \\
\text { buclizina (dicloridrato de) + carnitina + gaba + } \\
\text { lisina + complexo B, carbidopa + levodopa, } \\
\text { cartilagem de tubarão, ciclobenzaprina (cloridrato } \\
\text { de), cloroquina (dicloridrato de), cloroquina } \\
\text { (difosfato), clorpromazina (cloridrato de), }\end{array}$ \\
\hline
\end{tabular}




\begin{tabular}{|c|c|c|c|}
\hline Outras Classes & Outras Classes & & $\begin{array}{l}\text { deferoxamina (mesilato de), diaminodifenil } \\
\text { sulfona, dimeticona, diosmina + hesperidina, } \\
\text { dorzolamina (cloridrato de), escopolamina ou } \\
\text { hioscina (butillbrometo de), escopolamina } \\
\text { (butilbrometo de) + dipirona sódica, finasterida, } \\
\text { frutose + ác. ascórbico + tiamina + riboflavina + } \\
\text { piridoxina + nicotinamida, hidróxido de alumínio, } \\
\text { hidróxido de alumínio + hidróxido de magnésio + } \\
\text { dimeticona, isoflavona, isotretinoína, lactulose, } \\
\text { metimazol, metotrexato, metronidazol, minoxidil, } \\
\text { Passiflora incarnata + Crataegus oxycantha + } \\
\text { Salix alba, permanganato de cálcio, queratina + } \\
\text { Cassia acutifolia + enxofre sublimado + bitartarato } \\
\text { de potássio, salmeterol (xinafoato de) + } \\
\text { fluticasona (propionato de), sildenafil (citrato de), } \\
\text { tamoxifeno, trimebutina (maleato de), Valeriana } \\
\text { officinalis, zinco + cobre + selênio + beta-caroteno } \\
\text { + ac. ascórbico + tocoferol, medroxiprogesterona } \\
\text { (acetato de), testosterona base, tibolona }\end{array}$ \\
\hline $\begin{array}{c}\text { Medicamentos } \\
\text { errados/inexistentes }\end{array}$ & & 172 & 然 \\
\hline Total & Total & 1344 & 197 \\
\hline
\end{tabular}

${ }^{1}$ Fármaco não é mais comercializado.

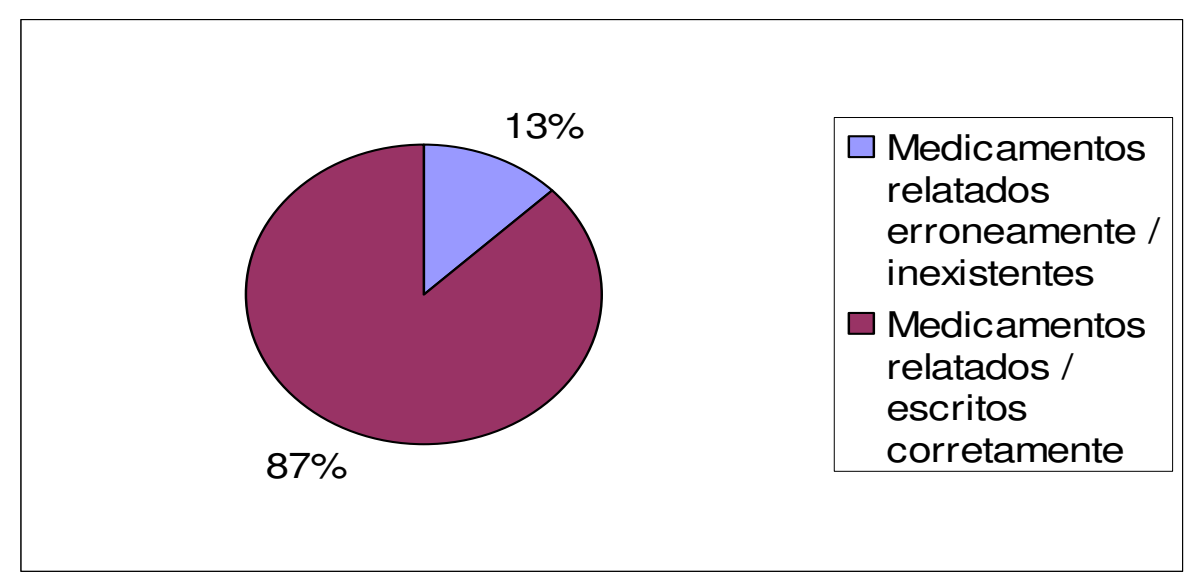

Figura 1. Representação da porcentagem de medicamentos relatados erroneamente/inexistentes.

Atualmente, pouca atenção é dirigida à detecção dos interferentes endógenos e exógenos, bem como aos efeitos causados pelos medicamentos, podendo levar a interpretações falseadas e incompatíveis com a real condição clínica do paciente (GIACOMELLI e PEDRAZZI, 2001). Por isso, uma maior atenção deve ser dada na coleta dos dados do paciente, principalmente quanto ao uso de fármacos, nos laboratórios de análises clínicas antes da realização dos exames. 


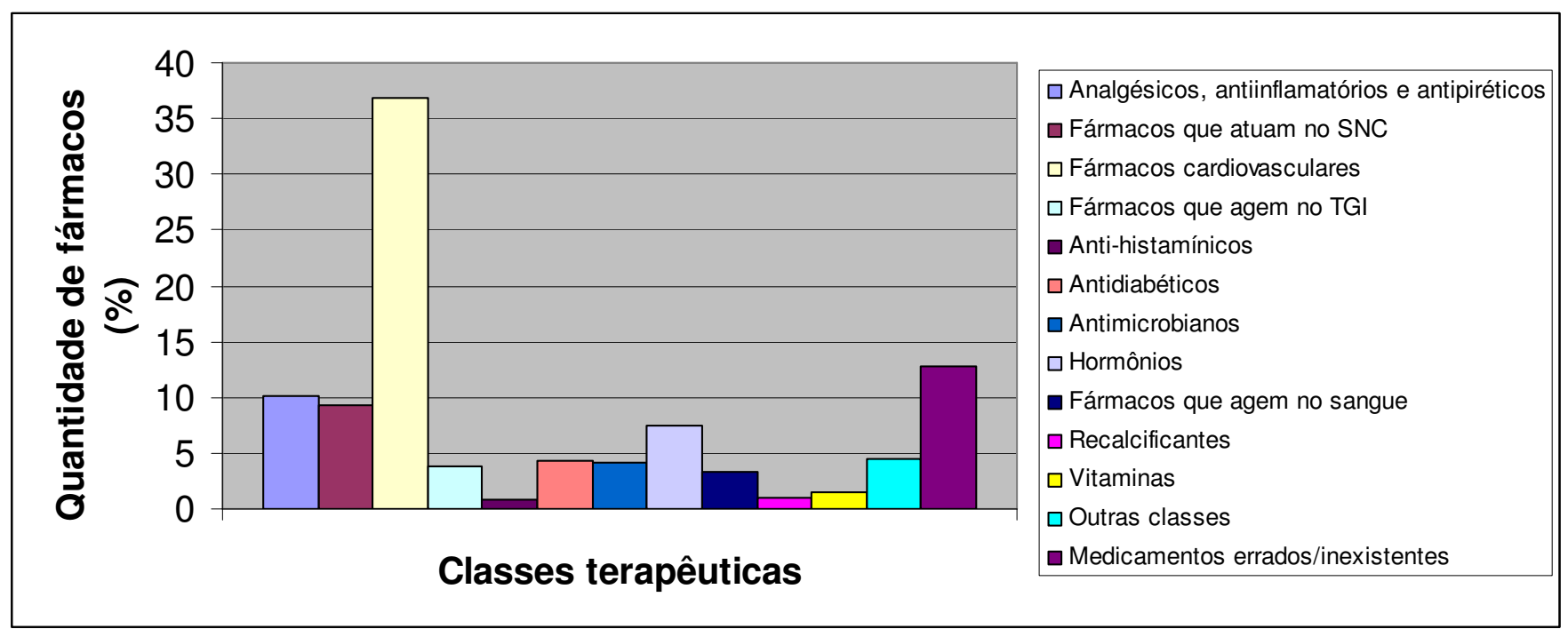

Figura 2. Representação da quantidade de fármacos de acordo com sua classe terapêutica.

Os fármacos foram divididos em classes terapêuticas como se observa na figura 2. Os fármacos cardiovasculares representam aproximadamente $37 \%$ do total, sendo estes os mais utilizados pelos pacientes do Centro de Análises Clínicas Rômulo Rocha. A alta incidência do uso destes fármacos justifica que dentre os cinco medicamentos mais utilizados pelos pacientes, quatro foram os selecionados para a discussão do presente artigo que pertencem a esta classe terapêutica. Assim, o captopril, o enalapril e o propranolol foram os anti-hipertensivos escolhidos e a hidroclorotiazida, o diurético de escolha.

Dentre os fármacos pesquisados foram selecionados os cinco mais utilizados (29\%): captopril, enalapril (maleato de), hidroclorotiazida, levotiroxina sódica e propranolol (cloridrato de) (Figura 3), para revisão profunda da literatura visando discussão a respeito de suas interações em testes laboratoriais. Esta discussão não foi feita utilizando as classes terapêuticas, pois dentro de uma mesma classe temos vários princípios ativos diferentes que podem interferir de maneira diferenciada nos exames.

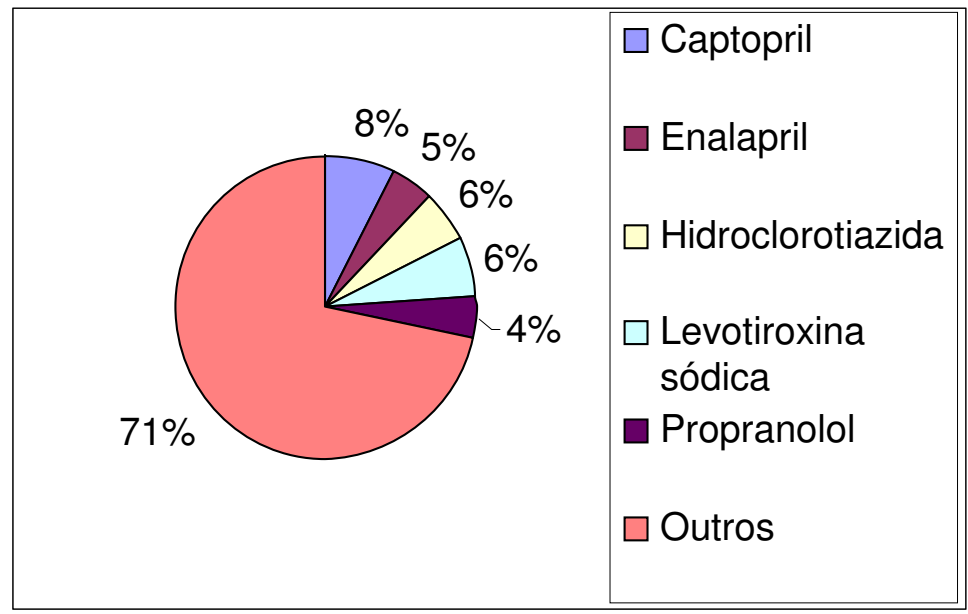

Figura 3. Representação da porcentagem dos cinco medicamentos mais utilizados pelos pacientes.

\section{Captopril}

O captopril pode proporcionar resultados falso-positivos para a detecção de cetonas na urina (YOUNG, 1995; ANVISA 2004/2005; COLOMBELI e FALKENBERG, 2006). Pode provocar hipercalemia (principalmente em pacientes com insuficiência renal) e hiponatremia (principalmente em pacientes sob dieta com restrição de sal ou sob tratamento concomitante com diuréticos), pode promover uma elevação transitória dos níveis de uréia e creatinina sérica principalmente em pacientes volume - ou sal - depletados ou com hipertensão renovascular (ANVISA, 2004/2005). Podem ocorrer também títulos positivos de anticorpos antinúcleo (FAN) e ainda elevações da aspartato aminotransferase, fosfatase alcalina e bilirrubina séricas nos pacientes em uso do captopril (YOUNG, 1995; ANVISA, 2004/2005). 
De acordo com a bula da marca Bayer Multistix ${ }^{\circledR} 10$ SG, o captopril também pode reduzir a sensibilidade do teste de hemoglobina em tira reagente para exame de urina (COLOMBELI e FALKENBERG, 2006).

O captopril pode ocasionar redução por efeito fisiológico no soro dos seguintes exames laboratoriais: enzima conversora de angiotensina (ECA), anticorpos anti-DNA, catecolaminas plasmáticas, proporção colesterol/HDL, imunoglobulina IgA e proporção LDL/HDL. Além disso, pode ocasionar redução por efeito fisiológico no plasma dos seguintes exames laboratoriais: angiotensina II, fator XI e fator XII. Em exames na urina, pode também provocar redução por efeito fisiológico na detecção da albumina, dopamina, hormônio antidiurético $(\mathrm{ADH})$, norepinefrina e osmolaridade urinária. No sangue pode causar diminuição dos eritrócitos, hematócrito, hemoglobina, leucócitos, neutrófilos e plaquetas (YOUNG, 1995).

O medicamento pode provocar aumento por efeito fisiológico dos seguintes exames: apolipoproteína AII, gama-glutamil-transferase (gama-GT), colesterol $\mathrm{HDL}_{3}$, desidrogenase lática (DHL) e uréia dosados no soro; prostaglandina $E_{2}$ na urina e renina no plasma (YOUNG, 1995).

Pode também ocorrer redução por efeito analítico no exame da enzima conversora de angiotensina (ECA) no soro e ainda um aumento na determinação de parâmetros bioquímicos séricos in vitro na dosagem de frutosamina (YOUNG, 1995).

\section{Enalapril (Maleato de)}

Fator antinúcleo (FAN) positivo e velocidade de hemossedimentação (VHS) elevada podem ser observados em pacientes que utilizam o enalapril (ANVISA, 2008).

Com a administração do enalapril foram observados aumentos de uréia sanguínea e creatinina sérica, elevação das enzimas hepáticas e/ou da bilirrubina sérica, geralmente reversíveis com a descontinuação do uso. Foram relatados também hipercalemia e hiponatremia (ANVISA, 2004/2005; BIOLAB FARMACÊUTICA, 2008). Há relatos de reduções de hemoglobina (YOUNG, 1995; ANVISA, 2004/2005) hematócrito, neutropenia, trombocitopenia, depressão da medula óssea e agranulocitose (ANVISA, 2004/2005).

Pode haver interferência do enalapril reduzindo a determinação dos seguintes parâmetros bioquímicos in vivo: aldosterona na urina; angiotensina II e insulina no plasma; enzima conversora de angiotensina (ECA), eritropoietina, desidrogenase lática $(\mathrm{DHL})$ e ácido úrico dosados no soro. $\mathrm{E}$ ainda, pode provocar um aumento por efeito fisiológico nos exames: amilase no soro; angiotensina I e renina no plasma; eosinófilos no sangue; Na/K ATPase nos eritrócitos e prostaglandina $E_{2}$ na urina (YOUNG, 1995).

\section{Hidroclotiazida}

A utilização da hidroclorotiazida aumenta a concentração da uréia in vivo (YOUNG et al., 1975; MOTTA, 1989; GIACOMELLI e PEDRAZZI, 2001).

Os diuréticos tiazídicos, como é o caso da hidroclorotiazida, podem causar um aumento nas dosagens de glicose e cálcio no sangue provocado por efeito fisiológico, assim como uma diminuição nos valores de potássio e sódio (MOTTA, 1989; HENRY, 1999). Estes também causam um aumento por efeito fisiológico nas dosagens sanguíneas de urato (ácido úrico). Já nos constituintes da urina, os diuréticos tiazídicos, podem causar uma diminuição, também por efeito fisiológico, nas dosagens de fenolsulfonftaleína, cetoesteróides (HENRY, 1999), cálcio, ácido úrico, cortisol e citrato; dosagem de cálcio nas fezes, granulócitos e neutrófilos dosados no sangue e a osmolaridade sérica também pode estar falsamente diminuída por efeito fisiológico (YOUNG, 1995). Observa-se uma redução por efeito fisiológico nos testes de tolerância a glicose (curva glicêmica) (MOTTA, 1989; YOUNG, 1995).

Pode haver aumento por efeito fisiológico nos seguintes exames: alanina aminotransferase (ALT), amilase, aspartato aminotransferase (AST), bilirrubina, cálcio ionizável, bicarbonato (YOUNG, 1995) e gamaglutamil-transferase (gama-GT) (MOTTA, 1989; YOUNG, 1995) séricos; cloreto, estriol, glicose, iodeto, fosfato, pirofosfato, sódio e zinco dosados na urina; glucagon plasmático e dosagem de hemoglobina $A_{1 C}$ ou glicosilada no sangue (YOUNG, 1995).

Em virtude dos efeitos no metabolismo do cálcio, os tiazídicos podem interferir com os testes de função da paratireóide (ANVISA, 2004/2005).

\section{Levotiroxina sódica}

A levotiroxina pode provocar redução por efeito fisiológico nos exames: apolipoproteína $B$, fosfolípides, imunoglobilina IgA, LDL, proporção LDL/HDL, mono-amino-oxidase (MAO) e colesterol séricos; anidrase carbônica I em eritrócitos; estriol na urina; neutrófilos no sangue e norepinefrina no plasma. $E$ ainda provocar aumento por efeito fisiológico nos exames seguintes: alanina aminotransferase (ALT), gama-glutamil-tranferase (gama-GT), tiroxina $\left(\mathrm{T}_{4}\right)$, colesterol $\mathrm{HDL}_{2}$ séricos; taxa de metabolismo basal (TMB); 2-hidroxiestrona e 2-metoxiestrona, ambos dosados na urina e tempo de protrombina plasmática (YOUNG, 1995). 
Várias drogas de amplo uso na prática clínica, como o propranolol, bloqueiam a conversão periférica de $\mathrm{T}_{4}$ para $\mathrm{T}_{3}$ nos tecidos periféricos. Tem sido descrito um efeito discreto do propranolol no bloqueio periférico de $\mathrm{T}_{4}$ para $\mathrm{T}_{3}$, sem alteração nos níveis séricos de hormônio estimulante da tireóide (TSH). O propranolol afeta 0 metabolismo extratireoideo do hormônio tireoideano (GRAF e CARVALHO, 2002).

O metabólito do propranolol (4-OH-propranolol) eleva aparentemente bilirrubinas (SUMITA, et al., 2005).

O propranolol pode alterar alguns testes clínicos laboratoriais, provocando níveis elevados de uréia sanguínea em pacientes com severa doença cardíaca (ANVISA, 2004/2005), elevação de transaminases séricas (YOUNG, 1995; ANVISA, 2004/2005), fosfatase alcalina e desidrogenase lática (ANVISA, 2004/2005).

Além disso, o propranolol pode causar redução por efeito fisiológico nos seguintes exames laboratoriais: enzima conversora de angiotensina (ECA), lecitina colesterol aciltransferase e curva glicêmica (teste de tolerância a glicose) séricos; adenosina trifosfato e 2,3-difosfoglicerato nos eritrócitos; adenosina cíclica monofosfato no plasma e nas plaquetas; granulócitos, neutrófilos e hematócrito no sangue; ácido hidroclórico no liquido gástrico (afeta a estimulação da histamina); hidroxiprolina e melatonina na urina. $E$ ainda, pode provocar aumento por efeito fisiológico nos seguintes exames: apolipoproteína C-III, creatina fosfoquinase (CPK MB), tiroxina ( $\left.T_{4}\right)$ livre, pré-albumina séricos; hemoglobina $A_{1 c}$ ou glicosilada no sangue; teste de tolerância a insulina e norepinefrina plasmáticos (YOUNG, 1995).

Dentre os cinco medicamentos mais utilizados observou-se uma maior quantidade de interferências in vivo do que in vitro. Dos exames citados, os seguintes são realizados no Centro de Análises Clínicas Rômulo Rocha: cetonas, uréia, creatinina, anticorpos antinúcleo (FAN), aspartato aminotransferase, fosfatase alcalina, bilirrubina, enzima conversora de angiotensina (ECA), anticorpos anti-DNA, hemoglobina, catecolaminas plasmáticas, proporção Colesterol/HDL, imunoglobulina IgA, fator XI, fator XII, albumina, osmolaridade urinária, eritrócitos, hematócrito, leucócitos, neutrófilos, plaquetas, renina, gama-glutamil-transferase (gama-GT), desidrogenase lática (DHL), frutosamina, velocidade de hemossedimentação (VHS), insulina, eritropoietina, ácido úrico, amilase, eosinófilos, glicose, cálcio, potássio, cortisol, citrato, osmolaridade sérica, testes de tolerância a glicose (curva glicêmica), aminotransferase (ALT), amilase, aspartato aminotransferase (AST), cálcio ionizável, iodeto, fosfato, sódio, zinco, glucagon, hemoglobina $A_{1 C}$ ou glicosilada, apolipoproteína $B$, fosfolípides, imunoglobilina $\lg A$, LDL, colesterol, tiroxina $\left(T_{4}\right)$, tempo de protrombina, creatina fosfoquinase (CPK MB) e hidroxiprolina.

Quando tratamos do assunto interações medicamentosas em exames laboratoriais existem muitas classes de medicamentos, além destes que foram discutidos no presente artigo, que causam interferências de forma significativa em testes laboratoriais. Dentre essas classes podemos citar os suplementos vitamínicos, que recentemente tem-se observado um aumento no consumo dessas substâncias, dentre elas altas doses de vitamina $\mathrm{C}$, que é um potente agente redutor, e quando presente em amostras biológicas podem interagir com os constituintes dos reagentes químicos e interferir em alguma etapa química da análise, causando resultados falseados, pode também ocorrer interferência por efeito fisiológico ou por ambos mecanismos (MARTINELLO e SILVA, 2003).

Outra classe que deve ser citada são os anticoagulantes, por exemplo o uso de warfarin e fluconazol (antifúngico) concomitantemente pode aumentar os efeitos anticoagulantes, resultando em um aumento do tempo de protrombina. Em um estudo de interação, o fluconazol aumentou em 12\% o tempo de protrombina, após a administração de warfarin em voluntários sadios do sexo masculino. É recomendável uma cuidadosa monitoração de pacientes que estejam recebendo fluconazol e anticoagulantes cumarínicos (CENTRALX BULAS, 2008).

No caso de antidiabéticos como por exemplo a metformina, pode haver um resultado falso-positivo de cetonas na urina. Concentrações de colesterol total, LDL e triglicerídeos podem estar reduzidas em usuários de

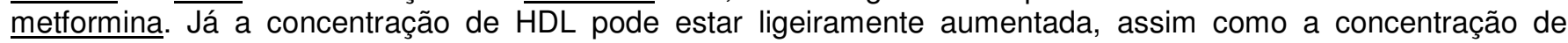
lactato no jejum (ANVISA, 2008).

O uso de aspirina, agentes antiinflamatórios não esteroidais (AINES) e anticoagulantes afetam a função plaquetária complicando o resultado de testes de função plaquetária e tempo de sangramento (HENRY, 1999).

\section{CONCLUSÕES}

Os resultados obtidos podem, também, ser utilizados para a promoção e planejamento de ações de Atenção Farmacêutica, já que é possível estratificar os tipos de doenças mais prevalentes a partir da terapêutica instituída.

As boas práticas em análises clínicas e toxicológicas são importantes para identificar, reduzir e/ou eliminar as fontes de erros potenciais no diagnóstico laboratorial. Para isso é necessária a educação continuada dos profissionais atuantes nas análises clínicas e toxicológicas (FERREIRA et al., 2007).

Constata-se que a profissão farmacêutica pode ser aperfeiçoada através da promoção da atenção farmacêutica, o que pode contribuir dignamente para o reconhecimento da profissão e para a manutenção da saúde dos pacientes (COSTA et al., 2006) de laboratórios, como do Rômulo Rocha e da população em geral.

Além disso, os profissionais que trabalham na recepção do laboratório devem ser devidamente treinados para que a coleta de informações sobre os medicamentos utilizados pelos pacientes seja feita de forma correta a fim de ajudar o técnico do laboratório na identificação dos mesmos e possíveis interações com os exames realizados. 
Pouca atenção é dirigida ao uso de vitaminas e essas são comumente utilizadas pela população. Devese estar atento para o uso de vitaminas pelo paciente, visto que muitas delas podem interferir em exames laboratoriais, como por exemplo, o ácido ascórbico que pode provocar resultados falso-negativos para dosagem de glicose, colesterol, triglicérides e ácido úrico (MARTINELLO e SILVA, 2003).

A melhoria da qualidade na prestação de serviços de saúde tem sido uma busca constante e cada vez mais crescente no país. Inúmeras variáveis podem interferir no desempenho da fase analítica e, conseqüentemente, na exatidão e precisão dos resultados dos exames, vitais para a conduta médica e, em última instância, para o bem-estar do paciente. Todos os laboratórios querem atender melhor e encantar o cliente. Ser atendido com excelência também é um desejo de todos. A difusão do conhecimento é a premissa básica para se alcançar estes objetivos (SUMITA et al., 2005).

\section{REFERÊNCIAS BIBLIOGRÁFICAS}

ANVISA - AGÊNCIA NACIONAL DE VIGILÂNCIA SANITÁRIA. Compêndio de Bulas de medicamentos. Vol. I (AG) / II (H-Z). Ed. ANVISA. 2004/2005.

ANVISA - BULÁRIO ELETRÔNICO [on line]. Disponível: http://bulario.bvs.br/index.php [capturado em 11 abril 2008].

BIOLAB FARMACÊUTICA [on line]. Disponível: http://www.biolabfarma.com.br/ [capturado em 06 abril 2008].

BRASIL. Conselho Federal de Farmácia. Resolução - RE no 357 de 27 de abril de 2001. Aprova o regulamento técnico das Boas Práticas de Farmácia. 2001.

CENTRALX BULAS [on line]. Disponível: http://www.bulas.med.br/ [capturado em 13 maio 2008].

COLOMBELI, A. S. S.; FALKENBERG M. Comparação de bulas de duas marcas de tiras reagentes utilizadas no exame químico de urina. Jornal Brasileiro de Patologia e Medicina Laboratorial. v. 42, n. 2, p. 85-93, 2006.

COSTA L. F.; SOUSA, L. G.; OLIVEIRA, A. M.; FONSECA C. A.; Atenção Farmacêutica para portadores de cuidados especiais. Revista Eletrônica de Farmácia, Supl. Vol 3 (2), p. 19-21, 2006.

DICIONÁRIO DE ESPECIALIDADES FARMACÊUTICAS - DEF. Rio de Janeiro: Editora de Publicações Científicas Ltda. 34를 Edição, 2005.

FACULDADE DE FARMÁCIA - UFG [on line]. Disponível: http://www.farmacia.ufg.br/page.php [capturado em 10 outubro 2007].

FERREIRA, M. M.; NETO, L. M. R.; PORTO, L. R. D.; MARTINS, J. B. J.; OLIVEIRA, G. S. L. O.; Análises Clínicas e toxicológicas. Conselho Regional de Farmácia do Estado de São Paulo, 2007.

GIACOMELLI, L. R. B.; PEDRAZZI, A. H. P. Interferência dos medicamentos nas provas laboratoriais de função renal. Arquivos de Ciências da Saúde da Unipar, p. 79-86, 2001.

GRAF H.; CARVALHO G. A. Fatores Interferentes na Interpretação de Dosagens Laboratoriais no Diagnóstico de Hiper e Hipotireoidismo. Arquivos Brasileiros de Endocrinologia \& Metabologia. v. 46, n.1, São Paulo, 2002.

HENRY, J. B. Diagnósticos Clínicos e Tratamento por Métodos Laboratoriais, 19a edição, São Paulo: Editora Manole Ltda, 1999.

MARTINELLO, F.; SILVA, E. L.; Interferência do ácido ascórbico nas determinações de parâmetros bioquímicos séricos: estudos in vivo e in vitro. Jornal Brasileiro de Patologia e Medicina Laboratorial. Rio de Janeiro, v. 39, n. 4, p. 323-334, 2003.

MOTTA, V. T. Bioquímica Clínica Métodos e Interpretações. 2ª edição, Porto Alegre: Editora Médica Missau, 1989.

MOTTA, V. T. Bioquímica Clínica Para o Laboratório Princípios e Interpretações. 4ª edição, Porto Alegre: Editora Médica Missau, 2003.

PEREIRA, L. R. L.; VECCHI, L. U. P.; BAPTISTA, M. E. C.; CARVALHO, D.; Avaliação da utilização de medicamentos em pacientes idosos por meio de conceitos de farmacoepidemiologia e farmacovigilância. Ciência \& Saúde Coletiva, 9(2), 479-481, 2004.

SILVA, C. M. S.; SOUZA, M. V. L. Hipotiroidismo autoimune refratário a altas doses de levotiroxina e hipocalcemia grave. Arquivos Brasileiros de Endocrinologia \& Metabologia., vol.49, №.4, São Paulo, 2005.

SUMITA N. M.; BARBOSA I.; ANDRIOLO, A.; CANÇADO, A. L.; VIEIRA, L. M. F.; MENDES, M. E.; ROMANO, P.; CASTRO, R. C.; OLIVEIRA, U. M. Recomendações da Sociedade Brasileira de Patologia Clínica / Medicina Laboratorial para Coleta de Sangue Venoso. 1 ${ }^{a}$.ed. Elaborado pelo Comitê de Coleta de Sangue da SBPC/ML e BD Diagnostics - Preanalytical Systems. 76 p. São Paulo, 2005. 
TRINDADE, I. S.; HEINECK, G.; MACHADO, J. R.; AYZEMBERG, H.; FORMIGHIERI, M.; CRESTANI, M.; GUSSO, J. Prevalência da Hipertensão Arterial Sistêmica na População Urbana de Passo Fundo (RS). Arquivos Brasileiros de Cardiologia. vol. 71 (no 2), 127-130, 1998.

(a) YOUNG, D. S. Effects of drugs on clinical laboratory tests. $4^{\text {a }}$ Ed. Washington: AACC Press, 1995.

(b) YOUNG, D. S.; PESTANER, L. C.; GIBBERMAN, V. Effects of drugs on clinical laboratory tests. Clin. Chem, 21(5):1D-432D, 1975. 